\title{
Evaluation of fast spin echo MRI sequence for an MRI guided high intensity focused ultrasound system for in vivo rabbit liver ablation
}

\author{
Nicos Mylonas ${ }^{1,2}$, Kleanthis Ioannides ${ }^{3}$, Venediktos Hadjisavvas ${ }^{1,4}$, Dimitris Iosif ${ }^{2}$, \\ Panayiotis A. Kyriacou ${ }^{1}$, Christakis Damianou ${ }^{2,4}$
}

${ }^{1}$ City University, London, UK;

${ }^{2}$ Frederick University, Limassol, Cyprus;

${ }^{3}$ Polikliniki Ygia, Limassol, Cyprus;

${ }^{4}$ MEDSONIC LTD, Limassol, Cyprus.

Email: dalma@cytanet.com.cy; venedik@,cytanet.com.cy; joseph.da@cytanet.com.cy; aktinodiagnostis@yahoo.com; cdamianou@cytanet.com.cy

Received 11 December 2009; revised 28 December 2009; accepted 6 January 2010.

\begin{abstract}
The effectiveness of magnetic resonance imaging (MRI) to monitor thermal lesions created by High Intensity Focused Ultrasound (HIFU) in rabbit liver in vivo is investigated. The MRI sequences of T1weighted, and T2-weighted fast spin echo (FSE) were evaluated. The main goal in this paper was to find the range of repetition time (TR) and range of echo time (TE) which maximizes the contrast to noise ratio (CNR). An ultrasonic transducer operating at $2 \mathrm{MHz}$ was used, which is navigated using a positioning device. With T1W FSE the range of TR under which CNR is maximized ranges from 400 to $900 \mathrm{~ms}$. The maximum contrast measured is approximately 25 . With T2W FSE the range of TE that establishes maximum contrast is between $40 \mathrm{~ms}$ and $80 \mathrm{~ms}$, with CNR of approximately 14 . T1W FSE is much better than T2W FSE in detecting thermal lesions in liver. Both T1W and T2 W FSE were proven successful to image thermal lesions created by HIFU in rabbit liver in vivo.
\end{abstract}

Keywords: Ultrasound; Liver; MRI; Lesion; Ablation

\section{INTRODUCTION}

Surgical resection is considered the therapy of choice for liver cancer. However, the percentage of patients who are good candidates for surgery is low [1]. Surgical resection is only feasible in $10-20 \%$ of the patients resulting to 5-year survival rates in the region of $40 \%$ [1].

This work was supported by the Research Promotion Foundation (RPF) of Cyprus under the contract ERYAN/2004/1, ANABA $\Theta \mathrm{MI} \Sigma \mathrm{H} /$ ПАГІО/0308/05, and ЕПІХЕІРН $\Sigma$ ЕІ $2 /$ ЕФАРМ /0308/01.
Moreover, the incidence of new metastases after resection is high, and the success rate after multiple resections is low [1]. Because of the above disadvantages of surgical resection the development of several less invasive local ablative therapies for liver tumors is imperative. These approaches have included percutaneous ethanol injection [2], cryotherapy [3], radiofrequency [4], microwave [5], and laser ablation [6,7]. These local therapies have produced survival rates similar to those with surgical resection in the treatment of metastases [8], but unfortunately high local recurrence rate is also reported [9].

Therefore thermal ablation methods could possibly become a main treatment option for liver cancer, especially if recurrence rate is minimized. Another ablative method that could be used for liver cancer treatment is High Intensity Focused Ultrasound (HIFU). HIFU is the only non-invasive local therapy to be proposed to date. If HIFU is proven equivalent to surgical resection, this minimally invasive approach may be able to replace surgery as the treatment of choice.

A lot of work has been done in many directions since the 80's in the area of liver ablation using HIFU. The threshold of intensity that is needed to cause irreversible damage in liver, was suggested by Frizell et al. 1987 [10] and Frizell 1988 [11]. This information is very useful, because the intensity needed to create lesions was defined. The thermal effects of HIFU in liver were well documented by ter Haar et al. 1989 [12], and Sibile et al. 1993 [13]. In the two studies by Chen et al. 1993 [14], and Chen et al. 1999 [15], the effect of HIFU ablation in liver and cancerous liver using histology were analysed extensively. The effective delivery of HIFU protocols in real oncologigal applications of liver was achieved by implanting tumour cells in liver $[16,17,18,19,20]$. 
Since the 90's clinical work has been initiated for liver cancer. Vallancien et al. [21] treated two patients with solitary liver metastases prior to surgical resection. The team headed by $\mathrm{Wu}$ in 1999 reported a clinical study for treating 68 patients with liver malignancies [22]. The same group reported a clinical study with 474 patients with Hepatocelular Carcinoma (HCC) treated using HIFU in combination with transarterial chemo-embolisation [23]. HIFU ablation has also been used for palliation in 100 patients with advanced-stage liver cancer [24]. Following treatment, symptoms, such as pain and lethargy, were relieved in $87 \%$ of the patients.

Without an imaging system that allows for online monitoring of the deposition of ultrasound energy or the creation of induced lesion, it is impossible to predict the precise location of the HIFU beam, to monitor the temperature changes, or to control the deposited thermal dose. In the past, these major constraints limited the development of HIFU as a noninvasive surgical technique. In recent years, however, integration of HIFU with MRI, which allows high-sensitivity tumor detection and the ability to monitor temperature in real time, has increased the potentials of HIFU.

MRI-guided HIFU has generally been reserved for the treatment of uterine fibroids [25] and breast adenomas [26]. However, it is very likely that this mode of treatment monitoring and delivery will have a role in the treatment of liver tumours. Recently, a non-randomised clinical trial is under way [27] to assess the safety and efficacy of the MRI guided HIFU system ExAblate 2000 (InSightec, Haifa, Israel) in the treatment of liver tumours. It was reported that a small number of patients has been treated to date with promising results [27].

The first attempt to monitor the effect of HIFU using MRI in liver was reported by Rowland et al. 1997 [28], who demonstrated that monitoring of thermal lesions in liver is feasible. The MRI appearance of lesions in liver created using HIFU was also studied by Jolesz et al. 2004 [29] and Kopelman et al. 2006 [30].

In this paper the goal is to investigate the effectiveness of MRI to monitor therapeutic protocols of HIFU in rabbit liver in vivo. The two basic and most important MRI sequences of T1-weighted fast spin echo (FSE), and T2-weighted FSE are investigated. The goal was to create large lesions and use MRI to discriminate between liver tissue and lesion. With T1W FSE the signal intensity vs. repetition time (TR) is evaluated and based on this analysis, the contrast to noise ratio (CNR) is estimated, in order to find the range of TR that produces maximum contrast. Similarly for T2W FSE the range of echo time (TE) is found that maximizes the contrast. A spherically focused transducer operating at $2 \mathrm{MHz}$ was used, which is navigated inside MRI using an MRI compatible robot.

\section{METHODS}

\subsection{HIFU/MRI System}

Figure 1 shows the block diagram of the HIFU/MRI system which includes the following subsystems:

a) HIFU system, b) MR imaging, c) Positioning device (robot) and associate drivers, and d) MRI compatible camera.

\subsubsection{HIFU System}

The HIFU system consists of a signal generator (HP 33120A, Agilent technologies, Englewood, CO, USA), a RF amplifier (250 W, AR, Souderton, PA, USA), and a spherically shaped bowl transducer made from piezoelectric ceramic of low magnetic susceptibility (Etalon, Lebanon, IN, USA). The transducer operates at $2 \mathrm{MHz}$, has focal length of $10 \mathrm{~cm}$ and diameter of $5 \mathrm{~cm}$. The transducer is rigidly mounted on the MRI-compatible positioning system (MEDSONIC LTD, Limassol, Cyprus) which is described shortly.

\subsubsection{MRI Imaging}

The 3-d positioning device and the transducer were placed inside a MRI scanner (Signa $1.5 \mathrm{~T}$, by General Electric, Fairfield, CT, USA). A spinal coil (USA instruments, Cleveland, OH, USA) was used to acquire the MRI signal.

\subsubsection{Positioning Device/Robot Drivers}

The robot has been developed initially for three degrees-of-freedom, but it can be easily developed for 5 degrees of motion. Since the positioning device is placed on the table of the MRI scanner its height is around $55 \mathrm{~cm}$ (bore diameter of the MRI scanner). The length of the positioning device is $45 \mathrm{~cm}$ and its width $30 \mathrm{~cm}$. The weight of the positioning device is only $6 \mathrm{~kg}$ and therefore it can be considered portable. Figure 2 shows the schematic the positioning device illustrating the 3 stages, transducer, and coupling method. The

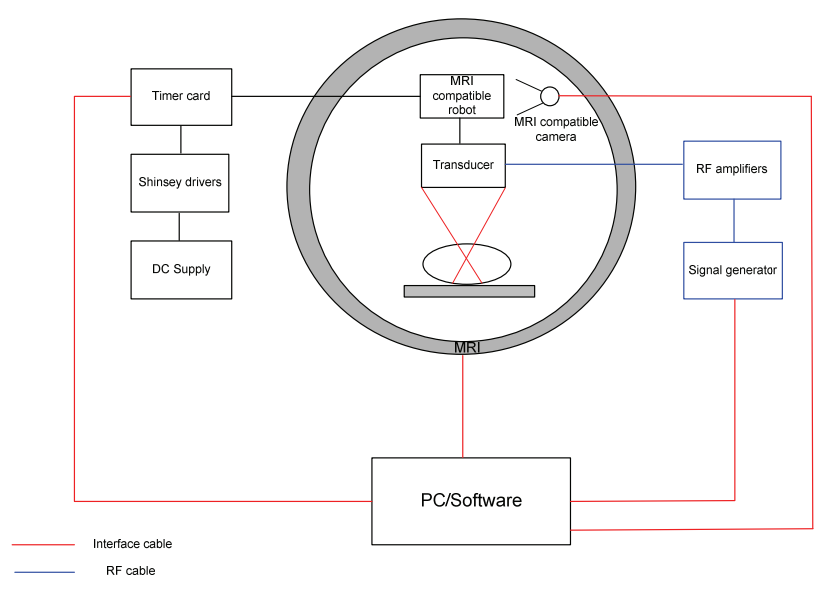

Figure 1. HIFU system under MRI guidance showing the various functionalities of the HIFU/MRI system. 


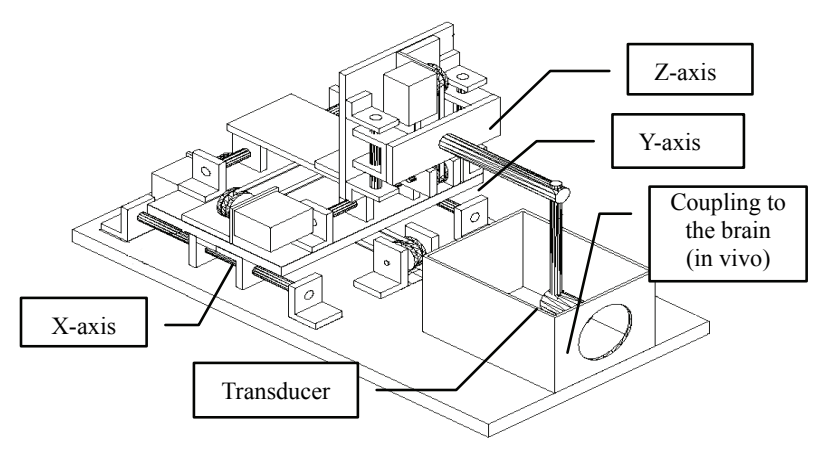

Figure 2. Schematic of the robot showing all of its stages.

positioning device operates by means of 3 piezoelectric motors (USR60-S3N, Shinsei Kogyo Corp., Tokyo, Japan). More details of this positioning device can be found in [31]. Moreover, the positioning system includes optoelectronic encoders (not shown in any of the figures) for providing signals indicating the relative positions of the movable elements in the positioning system. The resolution of all 3 axes of the positioning device is $0.1 \mathrm{~mm}$.

The box hosting the motor drivers is placed outside the MRI room since magnetic materials are involved. A DC supply $(24 \mathrm{~V}, 6 \mathrm{~A})$ is used to drive the Shinsei drivers. Wires from the Shinsei drivers are connected to a PCI 6602 interface card (National instruments, Austin, Texas, USA) via a connecting block. The PCI 6602 interface card includes timing and digital I/O modules. The interface is connected in a PC (Dell Inc. Round Rock, Texas, USA).

\subsubsection{MRI Compatible Camera}

In order to monitor the condition of the animal or humans (future use), an MRI compatible camera (MRC Systems GmbH, Heidelberg, Germany) was mounted on the system. The camera was interfaced by means of a video card. With the aid of the MRI compatible camera, the researcher can monitor the welfare of the animal.

\subsection{In Vivo Experiments}

For the in vivo experiments, New Zealand adult rabbits were used weighting approximately $3.5-4 \mathrm{~kg}$. Totally 7 rabbits were used in the experiments. The rabbits were anaesthetized using a mixture of $500 \mathrm{mg}$ of ketamine (100 mg/mL, Aveco, Ford Dodge, IA), $160 \mathrm{mg}$ of xylazine $(20 \mathrm{mg} / \mathrm{mL}$, Loyd Laboratories, Shenandoah, IA), and $20 \mathrm{mg}$ of acepromazine $(10 \mathrm{mg} / \mathrm{mL}$, Aveco, Ford Dodge, IA) at a dose of $1 \mathrm{~mL} / \mathrm{kg}$. The animal experiments protocol was approved by the national body in Cyprus responsible for animal studies (Ministry of Agriculture, Animal Services).

\subsection{HIFU Parameters}

The in situ spatial average intensity was estimated based on the applied power and the half-power width of the beam of the transducer. The attenuation used was $4 \mathrm{~Np} /$ $\mathrm{m}-\mathrm{MHz}$. The half-power length of the beam is $15.6 \mathrm{~mm}$ and the half-power width is $1.2 \mathrm{~mm}$. The details of the intensity estimation can be found in [32]. In order to create large lesions, a square grid pattern of $4 \times 4$ overlapping lesions was used. The spacing between successive transducer movements was $2 \mathrm{~mm}$, which creates overlapping lesions for the intensity and pulse duration used. In all the exposures the ultrasound was turn on for $5 \mathrm{~s}$. The in situ spatial average intensity used was 1000 $\mathrm{W} / \mathrm{cm}^{2}$. The delay between successive ultrasound firings was $10 \mathrm{~s}$.

\subsection{MRI Processing}

The following parameters were used for T1-W FSE: TR was variable from $100-1000 \mathrm{~ms}, \mathrm{TE}=9 \mathrm{~ms}$, slice thickness $=3 \mathrm{~mm}($ gap $0.3 \mathrm{~mm})$, matrix $=256 \times 256, \mathrm{FOV}=$ $16 \mathrm{~cm}, \mathrm{NEX}=1$, and $\mathrm{ETL}=8$. For T2-W FSE: TR $=$ $2500 \mathrm{~ms}$, TE was variable from $10 \mathrm{~ms}$ to $160 \mathrm{~ms}$, slice thickness $=3 \mathrm{~mm}($ gap $0.3 \mathrm{~mm})$, matrix $=256 \times 256$, $\mathrm{FOV}=16 \mathrm{~cm}, \mathrm{NEX}=1$, and $\mathrm{ETL}=8$.

The contrast to noise ratio (CNR) was obtained by dividing the signal intensity difference between the Region of Interest (ROI) in the lesion and in the ROI of normal liver tissue by the standard deviation of the noise in the ROI of normal liver tissue. The ROI was circular with diameter of $3 \mathrm{~mm}$.

The tissue temperature change $(\Delta T)$ has been estimated using the proton resonance frequency method given by the equation stated in Chung et al. 1996 [33]:

$$
\Delta \phi=\gamma B_{0} \alpha \Delta T \text { TE }
$$

where $\Delta \phi$ is the temperature-dependent phase shift which is the phase acquired before and during temperature elevation and which accumulates during the echo time TE using fast spoiled gradient (FSPGR). The other terms are $\gamma$ which is the gyromagnetic ratio of proton, $42.58 \mathrm{MHz} / \mathrm{T}, \alpha$ is the average proton resonance frequency coefficient, and $B_{0}$ is the flux density of the static magnetic field. The measured temperature elevation can be added to the base-line temperature to obtain the absolute temperature. The average proton resonance frequency coefficient $\alpha$ for the frequency shift was taken to be $-0.0105 \mathrm{ppm} /{ }^{\circ} \mathrm{C}$ as determined by the method described by Vykhodtseva et al. 2000 [33].

\section{RESULTS}

The goal in this study was to use T1W FSE using different TR (from 100 to $1000 \mathrm{~ms}$ ) and then evaluate the effect of TR on the CNR. Figure 3 shows a large lesion in liver in vivo using T1-w FSE (TR = $400 \mathrm{~ms}$ ). This lesion was created using in situ spatial average intensity 


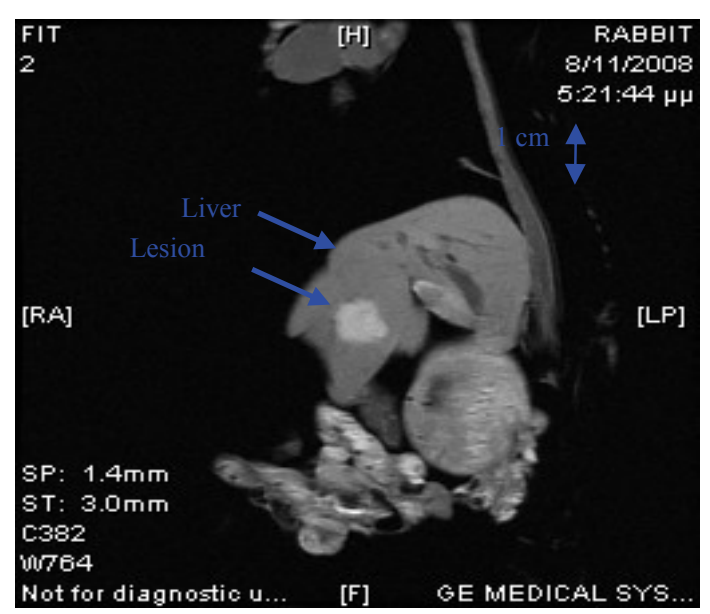

Figure 3. Large lesion in liver in vivo using T1-w FSE.

of $1000 \mathrm{~W} / \mathrm{cm}^{2}$ for $5 \mathrm{~s}$. Since the step size of this $4 \times 4$ lesion was $2 \mathrm{~mm}$, the size of this lesion is approximately $8 \mathrm{~mm} \times 8 \mathrm{~mm}$. The MRI estimated maximum temperature in this lesion was $65^{\circ} \mathrm{C}$. Since the estimated temperature is below $100^{\circ} \mathrm{C}$, the occurrence of boiling was excluded. The thermal lesion appears bright and the contrast with liver tissue is excellent.

Figure 4 shows the photograph of the lesion of Figure 3 after the animal was sacrificed in a plane perpendicular to the transducer face.

Figure 5 shows the CNR between lesion and liver plotted against TR for the MRI image of Figure 3. The same trend of CNR was seen in all the remaining 6 rabbits. Also the maximum CNR between liver and lesion of the other 6 rabbits was also close to 25 , and thus we are confident that this typical graph represents the behaviour of CNR vs. TR for rabbit liver ablation in vivo. The relaxation time $\mathrm{T} 1$ of the lesion is $250 \mathrm{~ms}$, and relaxation time $\mathrm{T} 1$ of the liver is $600 \mathrm{~ms}$. The proton density of the lesion increases by $20 \%$ compared to the host tissue.

Figure 6 shows the MRI image of the lesion of Figure 3 using T1-w FSE demonstrating the excellent propagation deep in the liver (i.e. in plane parallel to the transducer beam axis).

The second goal in this study was to explore T2W FSE using different TE (from 10 to $140 \mathrm{~ms}$ ) and then evaluate the effect of TE on the CNR. Figure 7 shows the MRI images of the same lesion as in Figure 3 using $\mathrm{T} 2 \mathrm{~W}$ FSE $(\mathrm{TE}=60 \mathrm{~ms})$.

Figure 8 shows the CNR between lesion and liver plotted against TE for the liver and lesion of the MRI image of Figure 7. The relaxation time T2 of lesion is 35 $\mathrm{ms}$ and the relaxation time $\mathrm{T} 2$ of the liver is $50 \mathrm{~ms}$. The proton density of the lesion decreases by $5 \%$ compared to the host tissue.

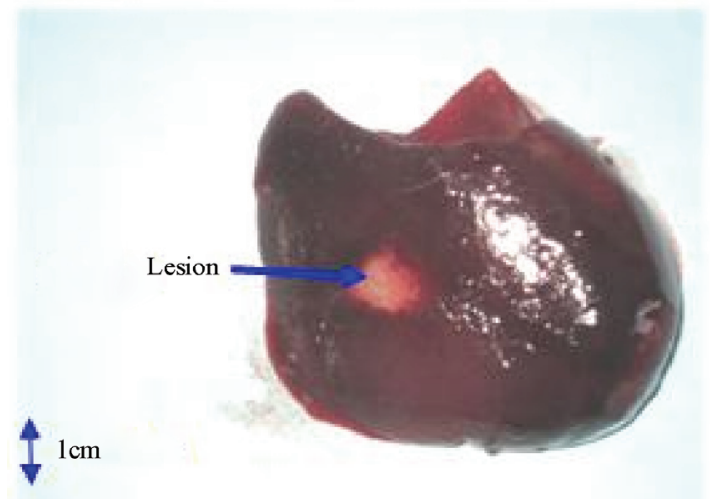

Figure 4. Photograph of the lesion of Figure 3.

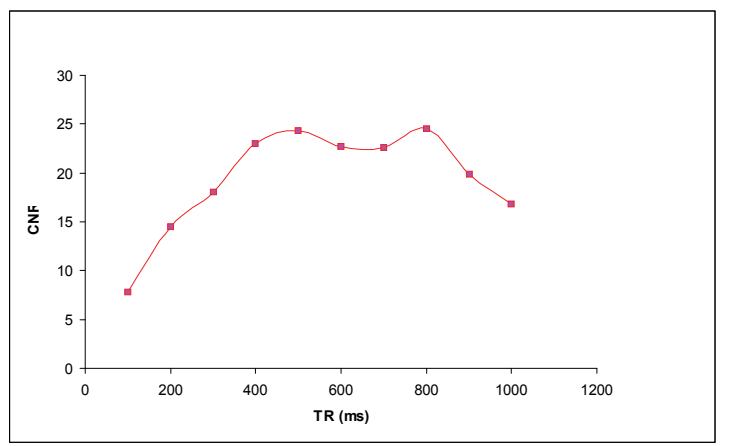

Figure 5. CNR vs TR.

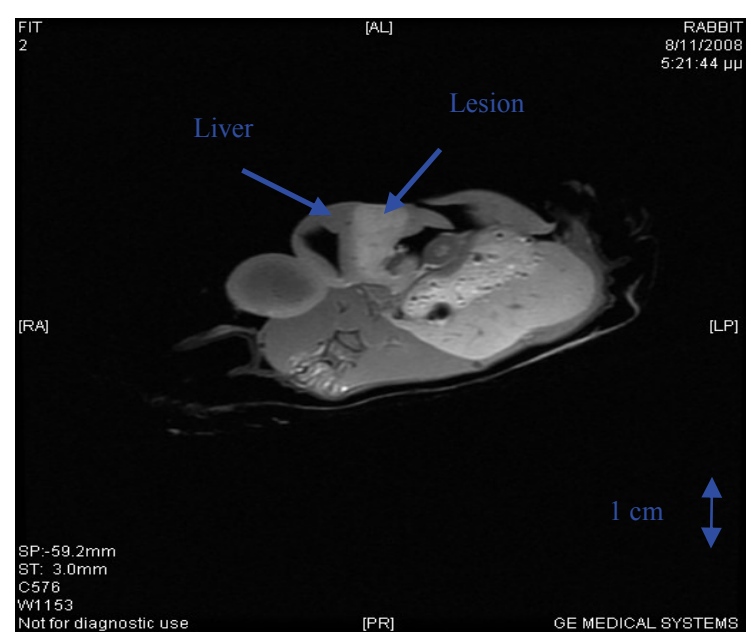

Figure 6. MRI image of the lesion of Figure 3 using T1W FSE.

\section{Discussion}

In this paper the goal was to measure the CNR of FSE MRI sequences in detecting thermal lesions created by HIFU in rabbit liver in vivo. Both T1-w FSE and T2-W FSE have been proven successfully for providing excellent contrast between liver and thermal lesion in rabbit in vivo.

The CNR with T1-w FSE is significantly higher than 


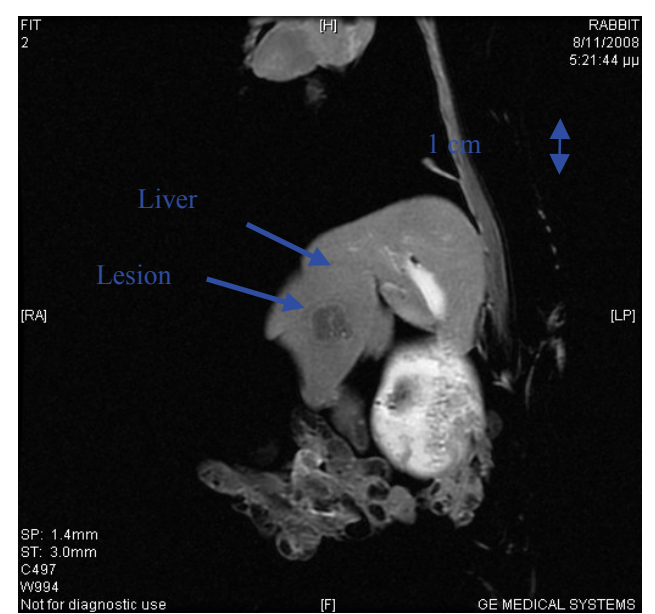

Figure 7. MRI images of the same lesion as in Figure 3 using T2W FSE.

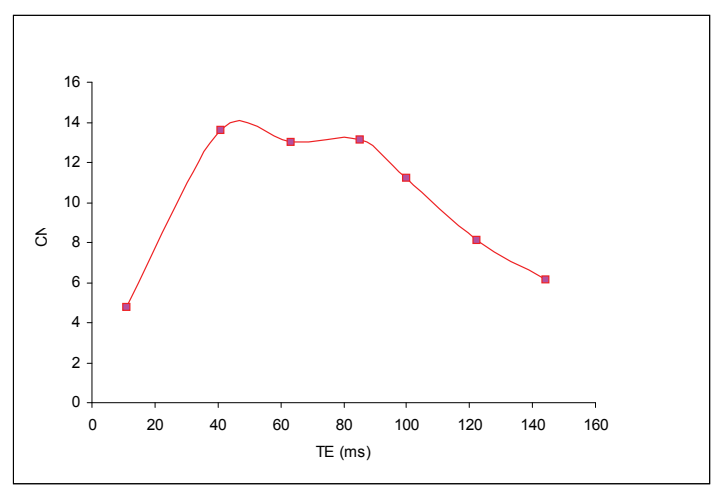

Figure 8. CNR vs TE for the MRI image shown in Figure 7.

T2-w FSE (25 with T1-w compared to 14 with T2-w). With T1W FSE the range of TR under which CNR is high and ranges from 400 to $900 \mathrm{~ms}$. Obviously one should use TR of $400 \mathrm{~ms}$ in order to minimize the imaging time. Thus, the optimum TR to be used is $400 \mathrm{~ms}$. The maximum contrast measured is approximately 25 .

The maximum CNR obtained for liver is the highest we measured after 17 years of experience in this field. The relaxation time $\mathrm{T} 1$ of lesion $(250 \mathrm{~ms})$ is much lower than the T1 of the host tissue (liver) which is $600 \mathrm{~ms}$. The greater the difference, the greater the CNR. However, one might not ignore the significant role that the value of proton density plays in the CNR. The proton density of the lesion is increased by $20 \%$.

The trend of CNR vs TR starts to increase then it becomes flat and then at high TRs it starts to decrease again. This trend is justified because at low TR, the difference in signal intensity between lesion and liver is low at the beginning and therefore CNR is lower. At higher TR the signal intensity of lesion and tissue reaches their maxima and therefore the signal difference is lower and hence the CNR drops again.

With T2-w the range of TE that establishes maximum contrast is between $40 \mathrm{~ms}$ and $80 \mathrm{~ms}$. This range was estimated by assuming that a CNR value of 10 is acceptable. Note that the maximum CNR value with T2-w FSE is around 14 which is much lower than the value obtained with T1-w FSE. The relaxation time T2 of lesion ( $35 \mathrm{~ms}$ ) is lower than the T2 of the host tissue (liver) which is $50 \mathrm{~ms}$. Therefore, in T2 W FSE the variation of signal intensity between lesion and liver is small (5\%) and therefore the factor dominating the CNR in T2-w FSE is the T2 relaxation time. The trend of CNR vs TE starts to increase then it becomes flat and then at high TEs it starts to decrease again. The same explanation holds as in the case of T1-w FSE.

\section{REFERENCES}

[1] Grasso, A., Watkinson, A. F., Tibballs, J. M. et al. (2000) Radiofrequency ablation in the treatment of hepatocellular carcinoma-clinical viewpoint. Journal of Hepatology, 33, 667-672.

[2] Livraghi, T., Lazzaroni, S., Meloni, F., Torzilli, G., and Vettori, C. (1995) Intralesional ethanol in the treatment of unresectable liver cancer. World Journal of Surgery, 19, 801-806.

[3] McCall, J. L., Booth, M. W., and Morris, D. L. (1995) Hepatic cryotherapy for metastatic liver tumors. British Journal of Hospital Medicine; 54, 378-381.

[4] Elias, D., De Baere, T., Smayra, T., Ouellet, J. F., Roche, A., and Lasser, P. (2002) Percutaneous radiofrequency thermoablation as an alternative to surgery for treatment of liver tumour recurrence after hepatectomy. British Journal of Surg, 89(6), 752-756.

[5] Yamanaka, N., Okamoto, E., Tanaka, T., Oriyama, T., Fujimoto, J., Furukawa, K., and Kawamura, E. (1995) Laparoscopic microwave coagulonecrotic therapy for hepatocellular carcinoma. Surg Laparosc Endosc, 5, 444449.

[6] Dick, E. A., Joarder, R., de Jode, M., Taylor-Robinson, S. D., Thomas H. C., Foster G. R., and Gedroyc, W. M. (2003) MR-guided laser thermal ablation of primary and secondary liver tumours, Clinical Radiology, 58(2), 112-120.

[7] Vogl, T. J., Straub, R., Eichler, K., Woitaschek, D., and Mack, M. G. (2002) Malignant liver tumors treated with MR imaging-guided laser-induced thermotherapy: Experience with complications in 899 patients $(2,520$ lesions). Radiology, 225(2), 367-377.

[8] Dodd, G. D., Soulen, M. C., Kane, R. A., Livraghi, T., Lees, W. R., Yamashita, Y., Gillams, A. R., Karahan, O. I., and Rhim, H. (2000) Minimally invasive treatment of malignant hepatic tumors: At the threshold of a major breakthrough. Radiographics, 20, 9-27.

[9] Solbiati, L., Livraghi, T., Goldberg, S. N., Ierace, T., Meloni, F., Dellanoce, M., Cova, L., Halpern, E. F., Gazelle, G. S. (2001) Percutaneous radio-frequency ablation of hepatic metastases from colorectal cancer: long-term results in 117 patients. Radiology, 221, 159-166.

[10] Frizzell, L., Linke, C., Carstensen, E., and Fridd, C. (1987) Thresholds for focal ultrasound lesions in rabbit kidney, liver and testicle. IEEE Transactions on Biomedical Engineering, 24(4), 393-396. 
[11] Frizzell, L. (1988) Threshold dosages for damage to mammalian liver by high intensity focused ultrasound. IEEE Transactions on Ultrasonics, Ferroelectrics and Frequency Control, UFFC-35, 578-581.

[12] Ter Haar, G., Sinnett, D., and Rivens, I. (1989) High intensity focused ultrasound-a surgical technique for the teatment of discrete liver tumors. Physics in Medicine and Biology, 34(11), 1743-1750.

[13] Sibille, A., Prat, F., Chapelon, J. Y., Fadil, F. A., Henry, L., Theillere, Y., Ponchon, T., and Cathignol, D. (1993) Extracorporeal ablation of liver tissue by high-intensity focused ultrasound. Oncology, 50(5), 375-379.

[14] Chen, L., Rivens, I., ter Haar, G., Riddler, S., Hill, C. R., and Bensted, J. P. (1993) Histological changes in rat liver tumours treated with high-intensity focused ultrasound. Ultrasound in Medicine \& Biology, 19(1), 67-74.

[15] Chen, L., ter Haar, G., Robertson, D., Bensted, J. P., and Hill, C. R., (1999) Histological study of normal and tumorbearing liver treated with focused ultrasound. Ultrasound in Medicine \& Biology, 25(5), 847-856.

[16] Yang, R., Reilly, C. R., Rescorla, F. J., Faught, P. R., Sanghvi, N. T., Fry, F. J., Franklin Jr, T. D., Lumeng, L., and Grosfeld, J. L. (1991) High-intensity focused ultrasound in the treatment of experimental liver cancer. $A r$ chives of surgery, 126(8), 1002-1210.

[17] Sibille, A., Prat, F., Chapelon, J. Y., abou el Fadil, F., Henry, L., Theilliere, Y., Ponchon, T., and Cathignol, D. (1993) Characterization of extracorporeal ablation of normal and tumor-bearing liver tissue by high intensity focused ultrasound. Ultrasound in Medicine \& Biology, 19(9), 803-813.

[18] Prat, F., Centarti, M., Sibille, A., Fadil, F. A., Henry, L., Chapelon, J. Y., and Cathignol, D. (1995) Extracorporeal high-intensity focused ultrasound for VX2 liver tumors in the rabbit. Hepatology, 21(3), 832-836.

[19] Cheng, S. Q., Zhou, Z. D., Tang, Z. Y., Yu, Y., Wang, H. Z., Bao, S. S., and Qian, D. C. (1997) High intensity focused ultrasound in the treatment of experimental liver tumour. Journal of Cancer Research and Clinical Oncology, 123(4), 219-223.

[20] Chen, L., ter Haar, G., Hill, C.R., Eccles, S. A., and Box, G. (1998) Treatment of implanted liver tumors with focused ultrasound. Ultrasound in Medicine \& Biology, 24(9), 1475-1488.

[21] Vallancien, G., Harouni, M., Veillon, B., Mombet, A., Prapotnich, D., Brisset, J. M., and Bougaran, J. (1992) Focused extracorporeal pyrotherapy: Feasibility study in man. Journal of Endourology, 6, 173-181.

[22] Wu, F., Chen, W., and Bai, J. (1999) Effect of high- intensity focused ultrasound on patients with hepatocellular cancer-preliminary report. The Chinese Journal of Ultra- sonography, 8, 213-216.

[23] Wu, F., Wang, Z. B., Chen, W. Z., Zou, J. Z., Bai, J., Zhu, H., Li, K.Q., Xie, F. L., Jin, C. B., Su, H. B., et al. (2004) Extracorporeal focused ultrasound surgery for treatment of human solid carcinomas: Early Chinese clinical experience. Ultrasound in Medicine \& Biology, 30, 245-260.

[24] Li, C. X., Xu, G. L., Jiang, Z. Y., Li, J. J., Luo, G. Y., Shan, H. B., Zhang, R., and Li, Y. (2004) Analysis of clinical effect of high-intensity focused ultrasound on liver cancer. World Journal of Gastroenterology, 10, 2201-2204.

[25] Stewart, E. A., Rabinovici, J., Tempany, C. M., Inbar, Y., Regan, L., Gostout, B., Hesley, G., Kim, H. S., Hengst, S., and Gedroyc, W. M. (2006) Clinical outcomes of focused ultrasound surgery for the treatment of uterine fibroids. Fertility and Sterility, 85(1), 22-29.

[26] Schmitz, A., Gianfelice, D., Daniel, B., Mali, W., and van den Bosch M. (2008) Image-guided focused ultrasound ablation of breast cancer: Current status, challenges, and future directions. European Radiology, 18, 1431-1441.

[27] Gedroyc, W. M. (2006) Magnetic resonance guided focused ultrasound (MRgFUS) treatment of liver tumours. In: Coussios, C.C., Ed., Proceedings of the 6th International Symposium on Therapeutic Ultrasound, AIP, Oxford.

[28] Rowland, I. J., Rivens, I., Chen, L., Lebozer, C. H., Collins, D. J., ter Haar, G. R., and Leach, M. O. (1997) MRI study of hepatic tumours following high intensity focused ultrasound surgery. British Journal of Radiology, 70, 144-153.

[29] Jolesz, F., Hynynen, K., McDannold, N., Freundlich, D., and Kopelman, D. (2004) Noninvasive Thermal Ablation of Hepatocellular Carcinoma by Using Magnetic Resonance Imaging-Guided Focused Ultrasound. Gastroenterology, 127, S242-S247.

[30] Kopelman, D., Inbar, Y., Hanannel, A., Freundlich, D., Castel, D., Perel, A., Greenfeld, A., Salamon, T., Sareli, M., Valeanu, A., and Papa, M. (2006) Magnetic resonanceguided focused ultrasound surgery (MRgFUS): Ablation of liver tissue in a porcine model. European Journal of Radiology, 59, 157-162.

[31] Damianou, C., Ioannides, K., and Milonas. (2008) Positioning device for MRI-guided high intensity focused ultrasound system. Computer-Assisted Radiology and Surgery, 2 (6), 335-345.

[32] Christakis, D., Pavlou, M., Velev, O., Kyriakou, K., and Trimikliniotis, M. (2004) High intensity focused ultrasound ablation of kidney guided by _RI. Journal of $\mathrm{Ul}$ trasound in Medicine and Biology, 30 (3), 397-404.

[33] Vykhodtseva, N., Sorrentino, V., Jolesz, F., Bronson, R. Hynynen, K. (2000) MRI detection of the thermal effects of focused ultrasound on the brain. Ultrasound in Medicine \& Biology, 26 (5), 871-880. 